\title{
Aurora-A/NF-kB Signaling Is Associated With Radio-resistance in Human Lung Adenocarcinoma
}

\author{
JUN-BAO LIU ${ }^{1 *}$, LIANG HU ${ }^{2 *}$, ZHIJIAN YANG ${ }^{3}$, YU SUN ${ }^{3,4}$, ROBERT M. HOFFMAN ${ }^{3,4}$ and ZHANG YI ${ }^{5}$ \\ ${ }^{1}$ Traditional Chinese Medicine Department, People's Hospital of Henan Province, \\ People's Hospital of Zhengzhou University, Zhengzhou, P.R. China; \\ ${ }^{2}$ Department of General Surgery, The Second Hospital of Nanjing, Nanjing \\ University of Chinese Medicine, Nanjing, P.R. China, \\ ${ }^{3}$ AntiCancer Inc., San Diego, CA, U.S.A.; \\ ${ }^{4}$ Department of Surgery, University of California, San Diego, CA, U.S.A.; \\ ${ }^{5}$ Department of Oncology, Jimin Hospital, Shanghai, P.R. China
}

\begin{abstract}
Background/Aim: This study aimed to discuss the effect and possible molecular mechanisms of Aurora-A/NF${ }_{k} B$ signaling on the radiotherapy resistance of human docetaxel-resistant lung adenocarcinoma cells. Materials and Methods: The human lung adenocarcinoma SPC-Al and $S P C-A 1 / D T X$ cell lines were utilized in the present study. The MTT assay measured the sensitivity of cells to radiotherapy. The tumor-initiating ability of the cells was detected in vitro by cloning assays. Apoptosis was quantified by flow cytometry. Real-time quantitative PCR and western blotting were used to detect the $m R N A$ and protein expression of the Aurora- $A / N F-k B$, respectively. Tumors transplanted subcutaneously into nude mice were used to test the effect of Aurora-A on the in vivo sensitivity of the tumors to radiotherapy. Results: The SPC-A1/DTX docetaxel-resistant lung adenocarcinoma cells were radio-resistant compared with the parental SPC-Al cells. Up-regulated aurora-A was responsible for the in vitro radio-resistance of docetaxelresistant SPC-AI/DTX cells. Nuclear transcription factor $N F-\kappa B$ was identified as a downstream target gene of Aurora-A in SPC-A1/DTX cells, and NF- $k B$ also participated in the radio-resistance of $S P C-A 1 / D T X$ cells regulated by Aurora-A. Conclusion: The Aurora-A/NF-kB pathway is association with radio-resistance of human lung adenocarcinoma docetaxel-resistant cells.
\end{abstract}

*These Authors contributed equally to this work.

Correspondence to: Zhang Yi, Department of Oncology, Jimin Hospital, Shanghai, 210000, PR China. Tel: +86 15021713798, e-mail: m15021713798@163.com

Key Words: Aurora-A, NF-kB, lung adenocarcinoma, radiotherapy.
Lung cancer is one of the leading causes of cancer-related death (1). Lung adenocarcinoma accounts for the main pathological types of lung cancer. Many patients are diagnosed at a late stage, losing the opportunity for surgery (2). Chemotherapy and radiotherapy are often used to improve the survival rate and prognosis of patients with advanced lung adenocarcinoma (3, 4). However, many patients with lung adenocarcinoma, especially those resistant to chemotherapy, are also resistant to radiotherapy (5). Therefore, it is important to understand the potential mechanisms of radio-resistant lung adenocarcinoma. In the present study, we generated a multidrug resistant strain of human lung adenocarcinoma, SPC-A1/DTX. The resistant cells also showed radio-resistance compared with the parental SPC-A1 cells. The present study aimed to further explore the potential molecular mechanisms of radio-resistance of human lung adenocarcinoma SPC-A1/DTX cells.

\section{Materials and Methods}

Cell line and chemical reagents. The following materials were used: NF-kB inhibitor (Selleck, Houston, TX, USA); Aurora-A interference plasmid (6) and docetaxel (Qi Lu Pharmaceutical, Jinan, PR China); antibodies against Aurora, NF-kB, IкBa, and GAPDH (Abcam, Cambridge, UK); fetal bovine serum (Sijiqing Co. Ltd., Hangzhou, PR China); RPMI-1640 culture medium (HyClone, Logan, UT, USA); a SYBR PrimeScript ${ }^{\mathrm{TM}}$ RT-PCR Kit (TaKaRa, Kusatsu, Japan); PVDF membrane (Millipore Co. Ltd, Billerica, MA, USA); human lung adenocarcinoma SPC-A1 cells (Shanghai Cell Research Institute, Shanghai, PR China).

Cell culture and transfection. Human lung adenocarcinoma SPC-A1 cells were cultured in RPMI-1640 medium containing 10\% FBS. Human lung adenocarcinoma SPC-A1/DTX cells were cultured in docetaxel at a concentration of $50 \mu \mathrm{g} / \mathrm{l}$. The culture conditions were $37^{\circ} \mathrm{C}, 5 \% \mathrm{CO}_{2}$, saturated humidity, with subculture every $48 \mathrm{~h}$. The Aurora-A interference plasmid was transfected into the cells using Lipofectamine 2000 according to the manufacturer's instructions. 
A

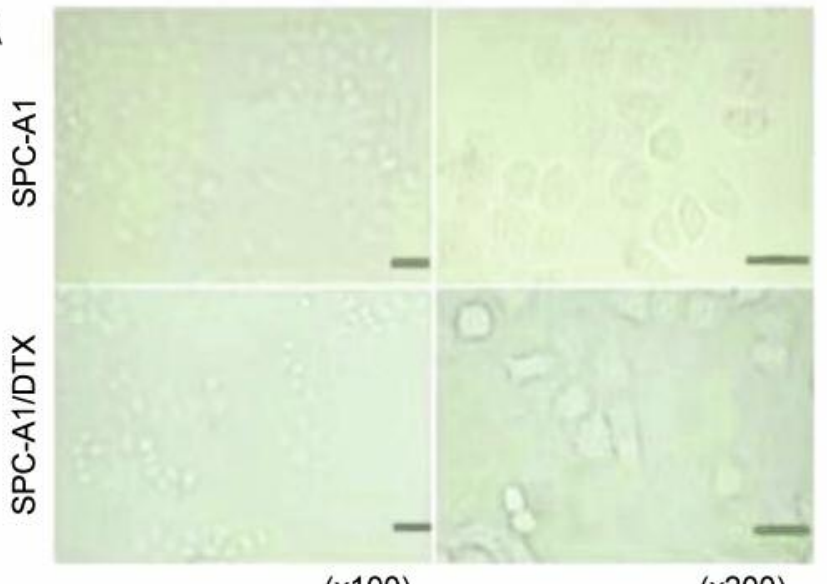

$(\mathbf{x} 100)$

$(\mathrm{x} 200)$
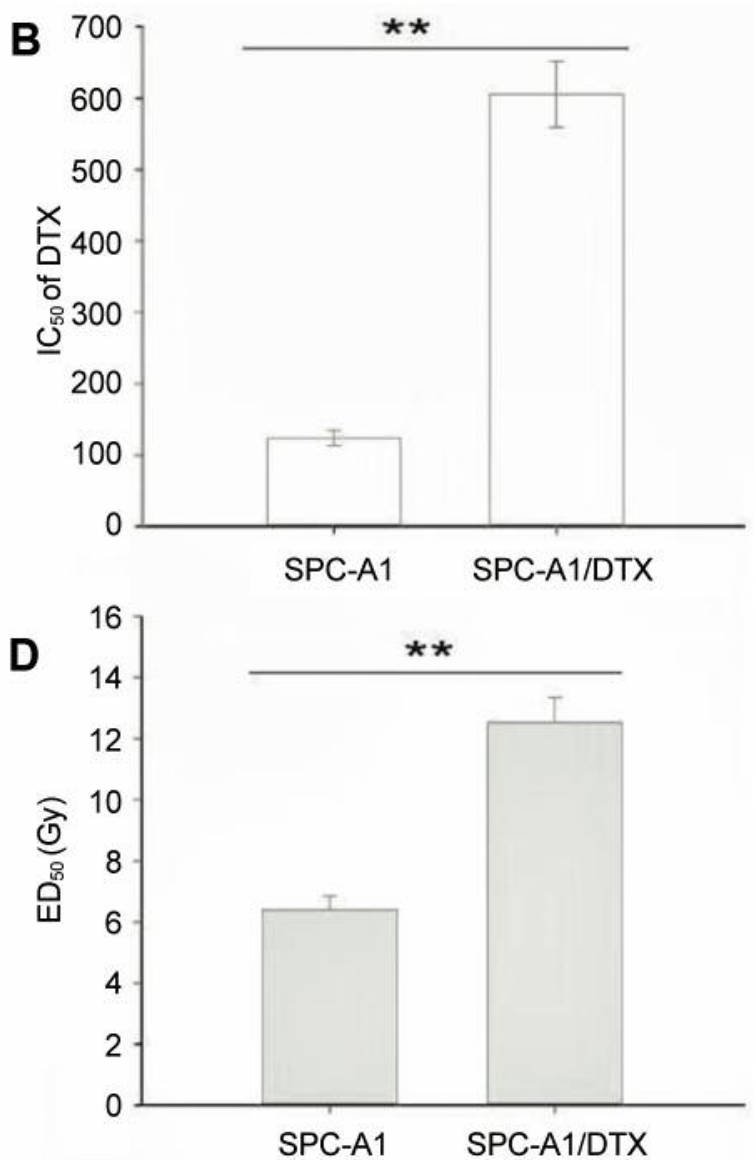

Figure 1. Multidrug-resistant human lung adenocarcinoma cells (SPC-A1/DTX) are radio-resistance. A. Morphology of human lung adenocarcinoma (LAD) parental cells (SPC-A1) and multidrug-resistant cells (SPC-A1/DTX) under a light microscopy. Upper: parental cells (SPC-A1); Lower: multidrug-resistant cells (SPC-A1/DTX). B. The MTT assay was used to calculate the $I_{50}$ value of docetaxel (DTX) for human LAD cells. C. The colony formation assay was conducted to estimate the proliferation rate of human LAD cells treated with different doses of irradiation. D. The MTT assay was used to calculate the $E D_{50}$ value of irradiation for human LAD cells. ${ }^{*} p<0.05,{ }^{* *} p<0.01$.

In vivo radiotherapy assay. Five-six weeks old $\mathrm{BALB} / \mathrm{c}$ athymic nude mice were provided by the Department of Comparative Medicine of the Jing Ling hospital. A total of $2.0 \times 10^{6}$ SPC-A1/DTX/ sh-control or SPC-A1/DTX/ sh-Aurora-A cells were suspended in $100 \mu \mathrm{l}$ PBS and transplanted subcutaneously into the right side of the posterior flank of male BALB/c athymic nude mice. Tumor volumes were calculated by using the equation: $\mathrm{V}=\mathrm{A} \times \mathrm{B} 2 / 2\left(\mathrm{~mm}^{3}\right)$, where $\mathrm{A}$ is the largest diameter and $\mathrm{B}$ is the perpendicular diameter. The tumorbearing nude mice were exposed to X-ray of 2.0 Gy alone when the average tumor size reached about $50 \mathrm{~mm}^{3}$. The treatment was repeated three times (the interval time was 5 days). After six weeks, all mice were sacrificed, and the primary tumors were excised, paraffin-embedded, formalin-fixed, stained with hematoxylin and eosin (H\&E) and immunostained for $\mathrm{Ki}-67$ and PCNA protein expression according to the manufacturer's instructions.

In vitro radio sensitivity assay. The in vitro radio sensitivity assay was determined with the MTT (3-4,5-dimethylthiazol-2-yl)-2,5diphenyltetrazolium bromide) assay in accordance with the manufacturer's instructions. Briefly, 2000 cells were plated in 96well plates $24 \mathrm{~h}$ after transfection. Next, the cells were treated with different doses of radiation and cultured for $48 \mathrm{~h}$. Then, MTT was added and incubated at $37^{\circ} \mathrm{C}$ for $4 \mathrm{~h}$, followed by the addition of DMSO to dissolve the resulting formazan crystal. The absorbance was detected at $490 \mathrm{~nm}$ with a microplate reader.

Cloning assay. One thousand cells were inoculated in 6-well culture plates. After 10-14 days of incubation, the cells were washed with PBS, fixed with pure methanol and stained with crystal violet.

Flow cytometric analysis. The early apoptosis rate of the cells was evaluated with the Annexin V/FITC-PI double-staining method. Briefly, digested cells were washed with precooled PBS. Then, $100 \mu \mathrm{l}$ of a working solution of propidium iodide (PI) and Annexin $\mathrm{V}$ was added to the cells. The cells were incubated in the dark for $15 \mathrm{~min}$, and stained cells were detected using flow cytometry (Becton Dickinson, NJ, USA).

Real-time quantitative RT-PCR. Total RNA was extracted using the Trizol reagent and reverse transcribed into cDNA with a SYBR Prime Script Kit (Takara Company, Shiga, Japan). Aurora-A primer was then added, and amplification was performed using the following reaction 
A

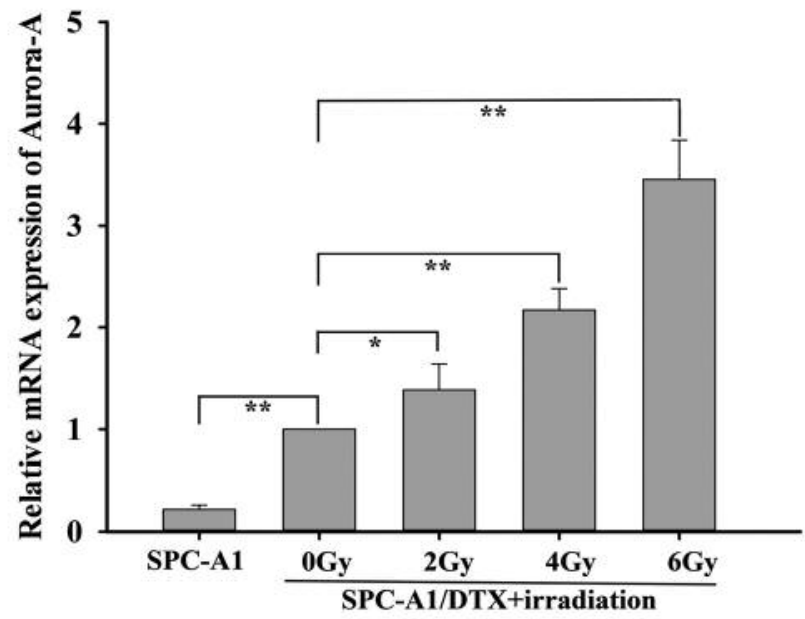

B

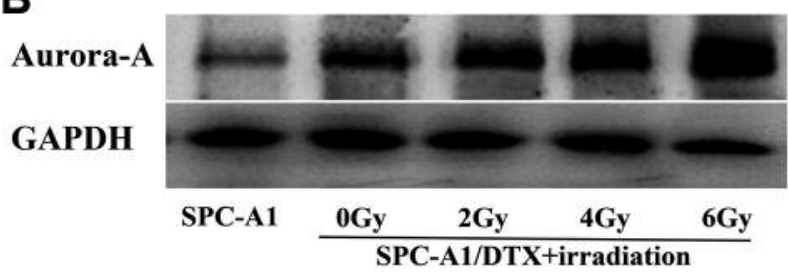

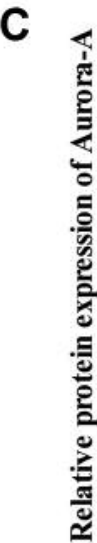

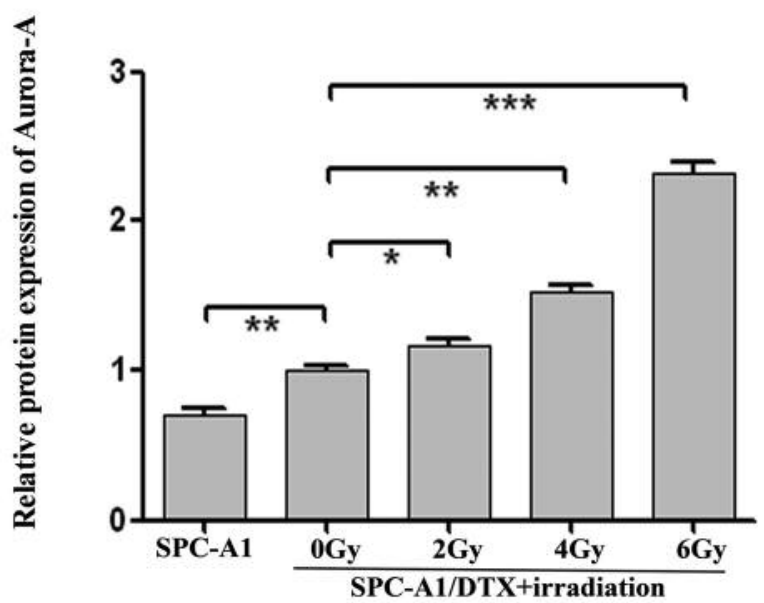

Figure 2. Irradiation up-regulates Aurora-A expression. A. The mRNA levels of Aurora-A were detected by real-time quantitative PCR in human LAD parental cells (SPC-A1) and multidrug-resistant LAD cells (SPC-A1/DTX) treated with different intensities of irradiation. GAPDH was used as a reference. $B-C$. The expression of Aurora-A protein was detected by western blotting in human LAD parental cells (SPC-A1) and multidrugresistant cells (SPC-AI/DTX) treated with different intensities of irradiation. GAPDH was used as a reference. The histogram represents the relative expression. $* p<0.05, * * p<0.01$.

conditions: $95^{\circ} \mathrm{C}$ pre-denaturation for 3 min, followed by 35 cycles of $94^{\circ} \mathrm{C}$ denaturation for $30 \mathrm{sec}, 55^{\circ} \mathrm{C}$ annealing for $30 \mathrm{sec}$, and $72^{\circ} \mathrm{C}$ extension for $60 \mathrm{sec}$. The expression of the target gene was detected using the Prism ABI 7000 Sequence Detection System. Gene expression was calculated using the $2^{-\Delta \Delta \mathrm{Ct}}$ method.

Western blotting. Total protein was extracted and separated by twelve percent sodium dodecyl sulfate-polyacrylamide gel electrophoresis (SDS-PAGE). The proteins were then transferred to a membrane, blocked with 5\% skim milk, and incubated with primary antibody at $4^{\circ} \mathrm{C}$ overnight. The membrane was then washed 3 times for 5 min each, incubated with secondary antibody, followed by 4 washes for $10 \mathrm{~min}$ each. Electrochemiluminescence was performed, and the Image $\mathbf{J}$ scanning gray value was evaluated.

Statistical methods. SPSS software (version 17.0) was used for the statistical analysis. The data were from three or more independent experiments. The data are shown as the mean \pm standard deviation. Two-group comparisons of quantitative data were analyzed by Student's $t$-test. $p$-Value $<0.05$ was considered statistically significant.

\section{Results}

Multidrug-resistant human lung adenocarcinoma SPC-A1/DTX cells show radiation resistance compared with parent $S P C-A 1$ cells. The human lung adenocarcinoma cell line SPC-A1/DTX, previously generated from parental human lung adenocarcinoma SPC-A1 cells, has multidrug resistance characteristics (7). Compared with the parental SPC-A1 cells, drug-resistant SPCA1/DTX cells were larger and multinucleated, and some cells had pseudopodia (Figure $1 \mathrm{~A}$ ). The $\mathrm{IC}_{50}$ value of docetaxel (DTX) in drug-resistant SPC-A1/DTX cells was significantly higher than that of parental cells (Figure 1B, $p<0.01$ ). In addition, we found that the proliferation rate of SPC-A1 cells was significantly decreased in vitro (Figure 1C). Furthermore, SPC-A1/DTX cells were more resistant to radiation than SPCA1 cells (Figure 1D, $p<0.01$ ). The above results suggested that the drug-resistant human lung adenocarcinoma SPC-A1/DTX cells also acquired radiation resistant.

Aurora-A is involved in the radiotherapy resistance of human lung adenocarcinoma drug-resistant SPC-A1/DTX cells. AuroraA is highly expressed in many kinds of malignant tumors. In the present study, we observed that the levels of Aurora-A mRNA and protein in the resistant strain were significantly higher than those in the parental strain (Figure 2A-C, $p<0.01$ ). Treatment with increased intensity of radiation, led to a further increase in the expression of Aurora-A in the resistant strain (Figure 2A-C). To further verify whether Aurora-A is involved in radiation 
A

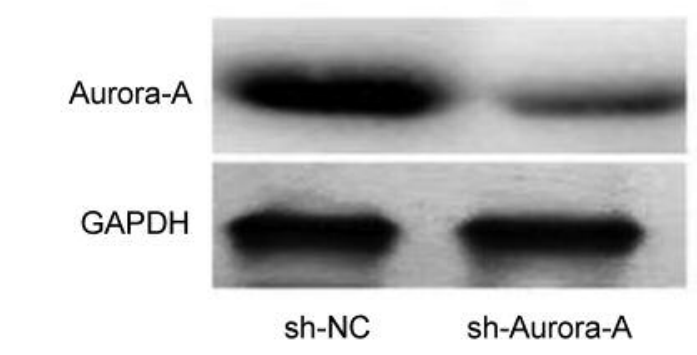

C

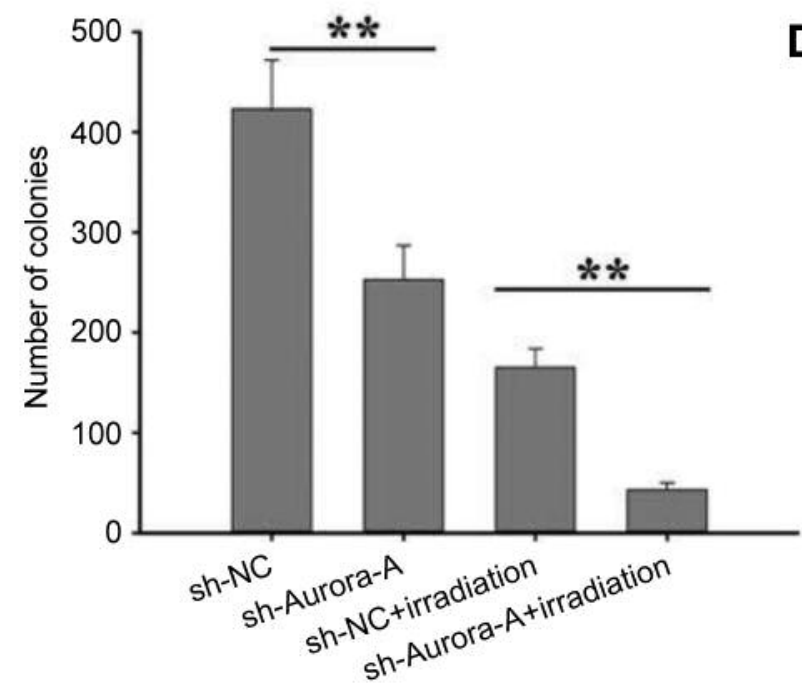

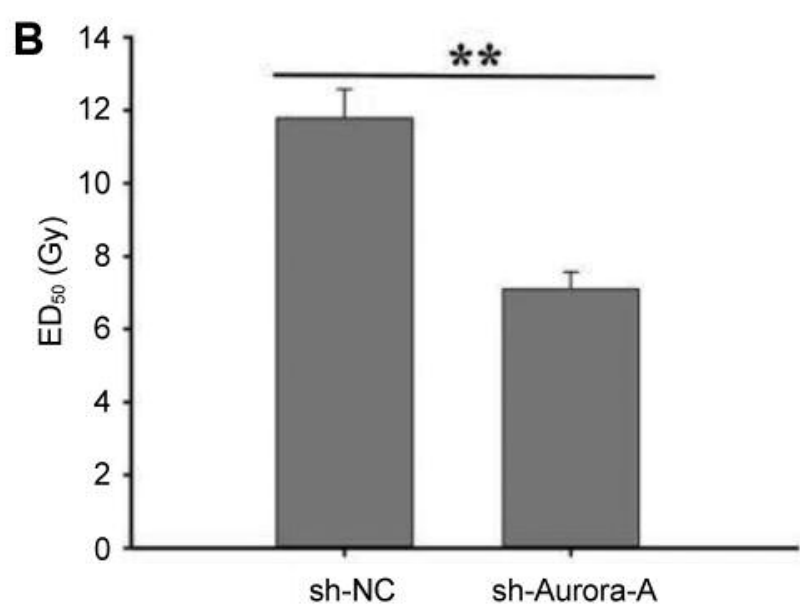

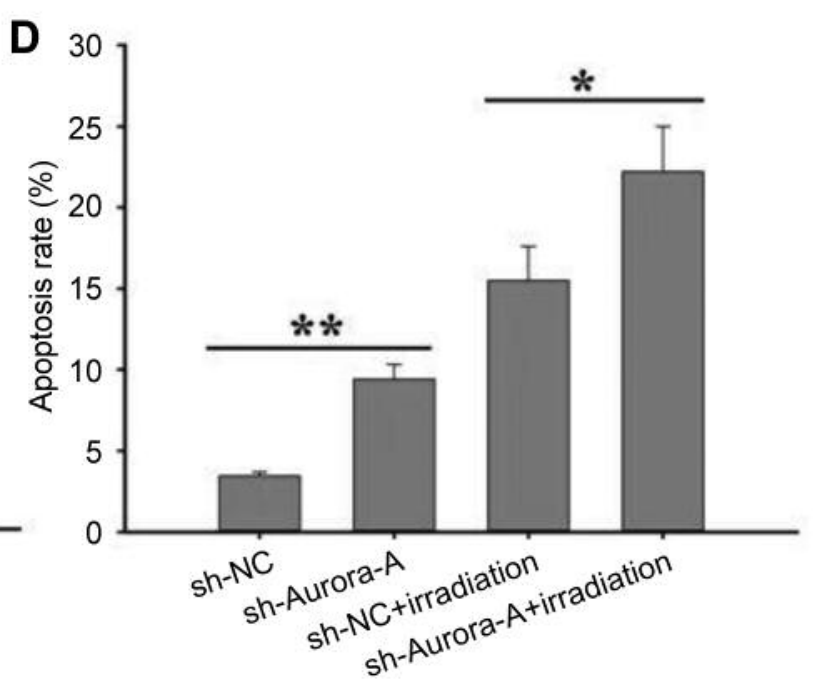

Figure 3. Down-regulation of Aurora-A expression can enhance the in vitro sensitivity of human LAD multidrug-resistant cells. A. Changes in Aurora-A protein expression in SPC-A1/DTX cells after transfection with small interfering RNA against Aurora-A. The expression levels of Aurora-A protein were detected by western blotting, and GAPDH was used as a reference. B. The ED $D_{50}$ of radiation of SPC-A1/DTX cells after transfection with small interfering RNA of Aurora-A. The ED 50 of each cell line was calculated with the MTT assay. C. The proliferation of SPC-A1/DTX cells in vitro following different treatments was determined by a colony-forming assay. Each cell type was plated in a 6-well plate with 1000 cells/well and was subjected to the corresponding treatment, crystal violet staining, image acquisition and calculation of the number of colonies after 2 weeks. D. The levels of early apoptosis of SPC-A1/DTX cells following different treatments was determined by flow cytometry. Each cell type was plated in a 6-well plate at 200,000 cells/well and was subjected to the corresponding treatment, and then, the rate of early apoptosis was determined by flow cytometry after $48 \mathrm{~h} . *^{*} p<0.05$, ** $p<0.01$.

resistance of human lung adenocarcinoma drug-resistant SPCA1/DTX cells, we constructed a small interfering RNA that targets Aurora-A, and the expression of Aurora-A protein was decreased after transfection (Figure 3A). Thereafter, we found that the effective dose (ED) 50 of radiotherapy for SPC-A1/DTX cells was significantly decreased following down-regulation of Aurora-A (Figure 3B, $p<0.01$ ). Irrespective of radiation treatment, down-regulation of Aurora-A expression inhibited the proliferation (Figure $3 \mathrm{C}, p<0.01$ ) and rate of early apoptosis (Figure 3D, $p<0.05$ ) of SPC-A1/DTX. The above results suggested that Aurora-A is involved in the radiation resistance of human lung adenocarcinoma.
Nuclear transcription factor $N F-k B$ is a downstream target gene of Aurora-A. Previous studies have suggested that Aurora-A can inhibit the expression of IkBa and cause the activation of $N F-k B$. However, whether this regulatory mechanism plays a role in human lung adenocarcinoma has not been reported. The present study found that downregulation of Aurora- $\mathrm{A}$ resulted in increased expression of $\mathrm{IkBa}$ and inhibition of the expression of NF-kB (Figure 4A, $p<0.01)$. Additionally, we found that the expression level of IkBa was decreased in the drug-resistant strain compared with the parental strain (Figure 4B). The expression level of NF$\mathrm{kB}$ was increased in the drug-resistant strain as the intensity 
A
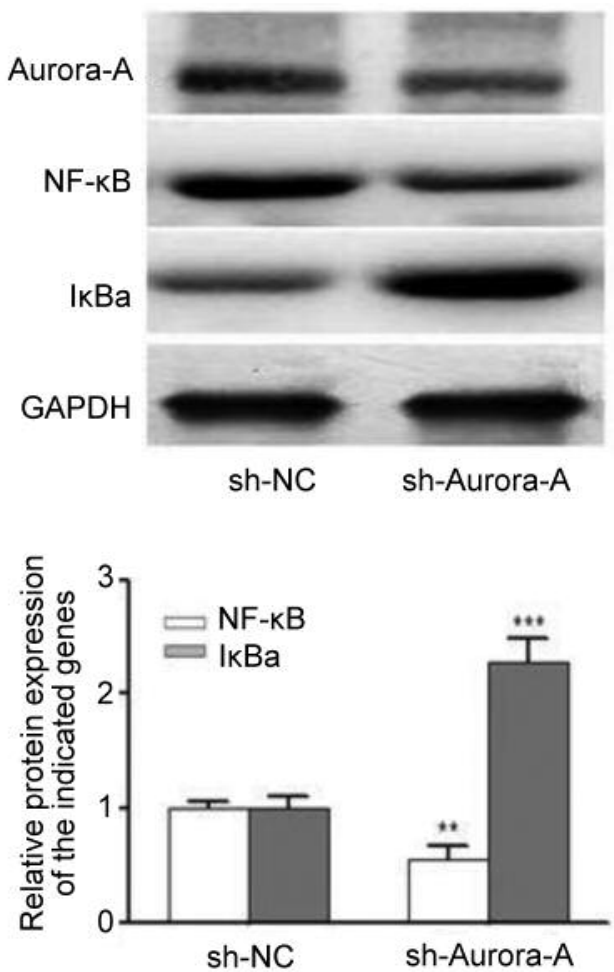

C

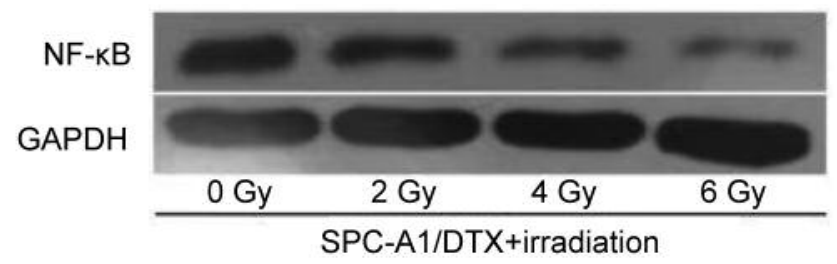

B
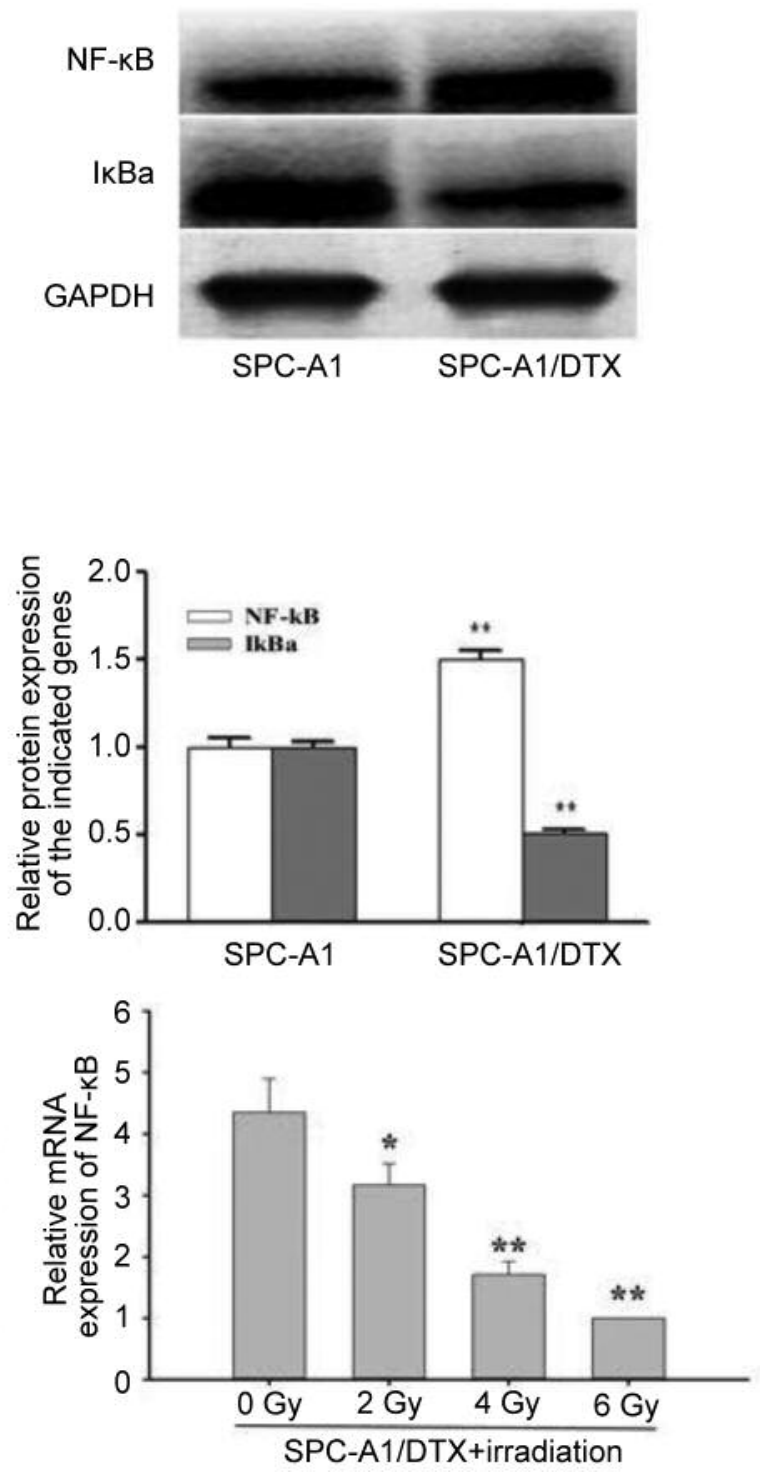

Figure 4. In drug-resistant SPC-Al/DTX cells, $N F-k B$ is the downstream target gene of Aurora-A. A. The expression levels of Aurora-A, $N F-k B$ and $I k B \alpha$ in SPC-Al/DTX cells after transfection with different small interfering RNAs. After $72 \mathrm{~h}$, the expression levels of Aurora-A, NF-kB and IkB $\alpha$ in each group was detected by western blotting, and GAPDH was used as a reference. The upper panel depicts representative western blots, and the lower panel represents the relative expression in a histogram. B. Expression levels of $N F-\kappa B$ and IkB $\alpha$ proteins in SPC-A1 and SPC-Al/DTX cells. The expression levels of $N F-K B$ and IKB $\alpha$ proteins in each group were detected by western blotting, and GAPDH was used as a reference. The upper panel depicts representative western blots, and the lower panel represents the relative expression in a histogram. $C$. The expression levels of $N F-k B$ protein in lung adenocarcinoma drug-resistant SPC-A1/DTX cells following treatment with different intensities of radiation. SPC-A1/DTX cells were treated with 0, 2, 4 and 6 Gy of radiation. After 48 h, the expression levels of $N F-k B$ protein were detected by western blotting, and GAPDH was used as a reference. The left panel depicts representative western blots, and the right panel represents the relative expression in a histogram. ${ }^{*} p<0.05, * * p<0.01$.

of the radiation dose increased (Figure 4C). These results indicated that nuclear transcription factor NF-kB is a downstream effector gene of Aurora-A in the human lung adenocarcinoma cell line SPC-A1/DTX.

$N F-\kappa B$ participates in the radiotherapy resistance of the human lung adenocarcinoma drug-resistant strain SPC-Al/DTX. To further verify that the downstream factor of Aurora-A, NF-kB, participates in radiation resistance of the human lung adenocarcinoma-resistant SPC-A1/DTX cells, we treated the cells with NF-kB inhibitor $(5 \mu \mathrm{mol} / \mathrm{l})$. We found that treatment of cells with the NF-kB inhibitor resulted in decreased expression of NF-kB protein (Figure $5 \mathrm{~A}$ ). The median $\mathrm{ED}_{50}$ was decreased after inhibition of NF-kB (Figure $5 \mathrm{~B}, p<0.01$ ). 

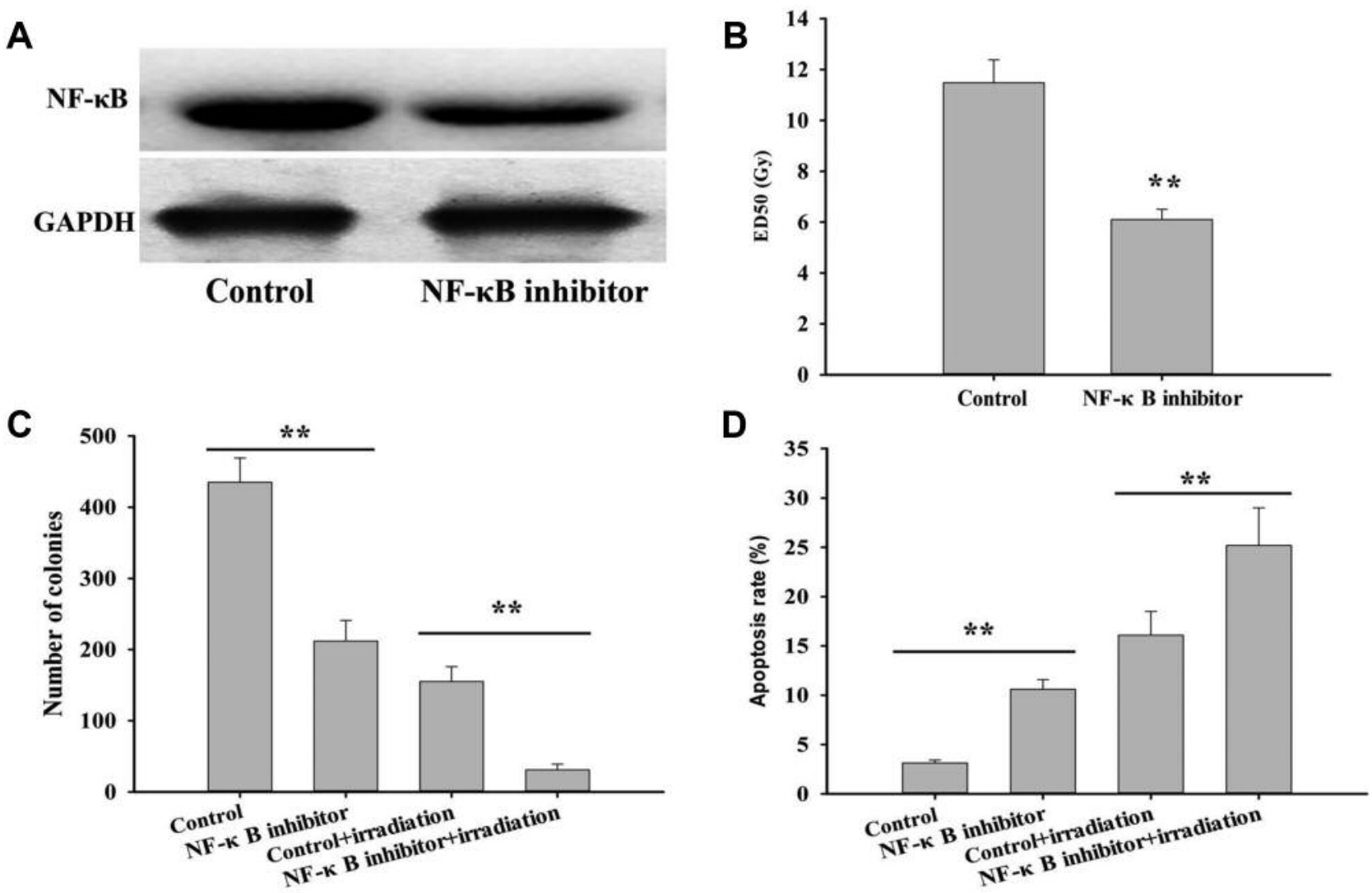

Figure 5. Inhibition of $N F-\kappa B$ can reverse the sensitivity to radiation of human lung adenocarcinoma drug-resistant SPC-A1/DTX cells. A. Changes in the $N F-K B$ protein expression in $S P C-A 1 / D T X$ cells after inhibiting $N F-K B$. The expression levels of $N F-k B$ protein was detected by western blotting, and GAPDH was used as a reference. $B$. The ED 50 of radiation in SPC-A1/DTX cells after inhibiting NF- $k B$. The ED 50 in each cell line was calculated by applying the MTT assay. C. The proliferation of SPC-A1/DTX cells in vitro was determined by the colony-forming assay. Each cell line was plated in a 6-well plate at 1000 cells/well and subjected to the corresponding treatment, followed by crystal violet staining, image acquisition and calculation of the number of colonies after 2 weeks. D. The levels of early apoptosis of SPC-A1/DTX cells were determined by flow cytometry. Each cell type was plated in a 6-well plate with 200,000 cells/well and subjected to the corresponding treatment, and then the rate of early apoptosis was determined by flow cytometry after $48 \mathrm{~h} .{ }^{*} p<0.05$, ** $p<0.01$.

Additionally, inhibition of NF-kB expression inhibited the proliferation of SPC-A1/DTX (Figure 5C, $p<0.01$ ) and increased the rate of early apoptosis with or without radiotherapy (Figure 5D, $p<0.01$ ). These results suggested that NF-kB is a downstream factor of Aurora-A in human lung adenocarcinoma.

Aurora-A is involved in the radiation resistance of human lung adenocarcinoma drug-resistant SPC-Al/DTX cells in vivo. To demonstrate the role of Aurora-A in radiation resistance of human lung adenocarcinoma drug-resistant SPC-A1/DTX cells in vivo, we subcutaneously implanted SPC-A1/DTX cells in nude mice. Compared with the control group, down-regulation of Aurora-A inhibited the growth of the subcutaneous tumors (Figure 6A, $p<0.05$ ). Following down-regulation of Aurora-A, the expression of Aurora-A and NF-kB in tumor tissue was decreased (Figure 6B). Furthermore, down-regulation of
Aurora-A also inhibited the proliferation of subcutaneously implanted tumor cells in nude mice (Figure $6 \mathrm{C}$ and $\mathrm{D}, p<0.05$ ).

\section{Discussion}

Aurora-A is a subtype of serine/threonine kinases that plays an important role in the normal process of cell mitosis and the occurrence and development of tumors (8). Aurora-A kinase is often over-expressed in various malignant tumors, such as lung cancer, esophageal cancer, liver cancer, breast cancer, and colon cancer, and it is related to the patient prognosis (9-13). It has also been reported that Aurora- $\mathrm{A}$ is involved in chemoresistance (14). Aurora-A is involved in chemotherapy sensitivity of hepatocellular carcinoma via the NF-kB/miR-21/PTEN/Akt signaling pathways. Overexpression of Aurora-A is closely related to the recurrence-free survival rate of patients. Aurora$\mathrm{A}$ is an independent prognostic factor in patients with 

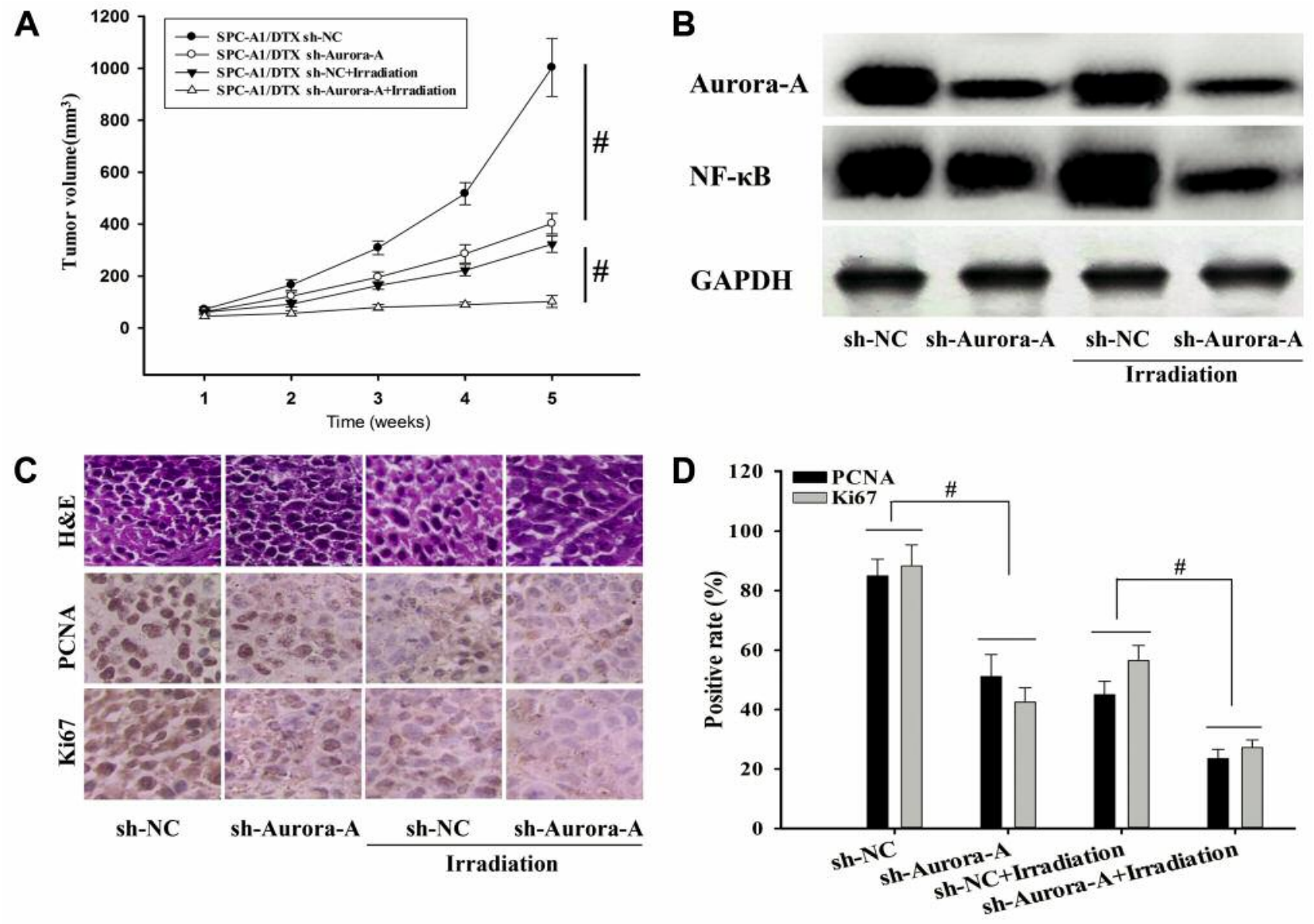

Figure 6. Down-regulation of Aurora-A expression can reverse the sensitivity to radiation of human lung adenocarcinoma drug-resistant SPCA1/DTX cells in vivo. A. Growth curves of the subcutaneously growing of SPC-A1/DTX cells tumors treated. The SPC-A1/DTX cells were transfected with Aurora-A small interfering RNA or the corresponding control. A tumor cells were subcutaneously implanted in nude mice $\left(2 \times 10^{6} /\right.$ each mouse, 4 miceleach group). The tumor size was measured every week, and all the mice were sacrificed after 5 weeks. Tumors were then removed, and growth curves were generated. B. The expression levels of Aurora-A and NF-kB proteins were changed in each group. The expression levels of Aurora-A and $\mathrm{NF}-\mathrm{kB}$ proteins in each group of tumors were detected by western blotting. GAPDH was used as a reference. $C$. H\&E staining, and PCNA and Ki67 expression. The expression levels of PCNA and Ki67 were determined by immunohistochemistry. D. The expression levels of PCNA and Ki67 were calculated in each experimental group. ${ }^{*} p<0.05$.

hepatocellular carcinoma (6). In non-small cell lung cancer, overexpression of Aurora-A is closely related to cisplatin resistance, lymph node metastasis and patient prognosis. The present study found that the expression of Aurora-A in drugresistant cells was significantly increased and down-regulation of Aurora-A could significantly reverse radio-resistance of human lung adenocarcinoma drug-resistant strain, suggesting that high levels of Aurora-A were involved in the radiation resistance of human lung adenocarcinoma drug-resistant SPCA1/DTX cells in vivo and in vitro. However, the underlying mechanism of the involvement of Aurora-A in radiation resistance has not yet been fully elucidated.

Previous studies have shown that Aurora-A decreases the stability of $\mathrm{IkB} \alpha$ in p53-knockout lung cancer, increasing the transcriptional activity of NF- $\mathrm{kB}$, which is involved in resistance to gefitinib (15). miR-15a/16 increased radiation sensitivity of non-small cell lung cancer by targeting the TLR1/NF- $\mathrm{kB}$ signaling pathway (16). NF-KB is not only an important factor regulating cell growth, differentiation, and inflammation but also an endogenous tumor-promoting factor. NF-kB binds to the pathway inhibitor protein IKB in the cytoplasm at resting state. Activation of NF- $\mathrm{KB}$ occurs primarily via the dissociation of ІкB or an increase in NF-kB expression. Radiotherapy can reduce the levels of NF-kB in tumor cells, and excessive activation of NF- $\mathrm{kB}$ can promote production of apoptosis-inhibiting factors and enhance tumor cell growth, resulting in radiation resistance (17-19). 
In the present study, we found that the expression of NF$\mathrm{KB}$ in the resistant strain was significantly higher than that in the parental strain, while the expression of IKB was decreased, and the expression of NF- $\mathrm{KB}$ was increased following down-regulation of Aurora-A. Radiation resistance can cause the expression of NF-KB to be further decreased. These results suggested that high levels of Aurora-A expression induce the activation of NF- $\mathrm{kB}$ that participates in the radiation resistance of human lung adenocarcinoma. In conclusion, the Aurora- $\mathrm{A} / \mathrm{NF}-\mathrm{kB}$ pathway is associated with radio-resistance of human lung adenocarcinoma docetaxel-resistant cells.

\section{Conflicts of Interest}

None of the Authors has a conflict of interest with regard to this study.

\section{Authors' Contributions}

Zhang Yi designed the study. Junbao Liu and Liang Hu performed the in vitro study. Yu Sun performed the in vivo study. Zhijian Yang and Robert M. Hoffman revised the manuscript.

\section{References}

1 Siegel RL, Miller KD and Jemal A: Cancer statistics. CA Cancer J Clin 68: 7-30, 2018. PMID: 29313949. DOI: 10.3322/caac.21442

2 Zhang K, Chen J, Chen D, Huang J, Feng B, Han S, Chen Y, Song H, De W, Zhu Z, Wang R and Chen L: Aurora-A promotes chemoresistance in hepatocellular carcinoma by targeting NF$\mathrm{kB} /$ microRNA-21/PTEN signaling pathway. Oncotarget 5: 1291612935, 2014. PMID: 25428915. DOI: 10.18632/oncotarget.2682

3 Wang R, Huang J, Feng B, De W and Chen L: Identification of ING4 (inhibitor of growth 4) as a modulator of docetaxel sensitivity in human lung adenocarcinoma. Mol Med 18: 874886, 2012. PMID: 22460125. DOI: 10.2119/molmed.2011.00230

4 Wan XB, Fan XJ, Chen MY, Xu J, Long ZJ and Hua YJ: Inhibition of Aurora-A results in increased cell death in 3dimensional culture microenvironment, reduced migration and is associated with enhanced radio sensitivity in human nasopharyngeal carcinoma. Cancer Biol Ther 8: 1500-1506, 2009. PMID: 19502819. DOI: $10.4161 / \mathrm{cbt} .8 .15 .8958$

5 Zheng X, Chi J and Zhi J: Aurora-A-mediated phosphorylation of LKB1 compromises LKB1/AMPK signaling axis to facilitate NSCLC growth and migration. Oncogene 37: 502-511, 2018. PMID: 28967900. DOI: 10.1038/onc.2017.354

6 Chen X, Cai S and Li B: Identification of key genes and pathways for esophageal squamous cell carcinoma by bioinformatics analysis. Exp Ther Med 16: 1121-1130, 2018. PMID: 30112053. DOI: 10.3892/etm.2018.6316

7 Wang LL, Jin XH and Cai MY: AGBL2 promotes cancer cell growth through IRGM-regulated autophagy and enhanced Aurora A activity in hepatocellular carcinoma. Cancer Lett 414: 71-80, 2018. PMID: 29126912. DOI: 10.1016/j.canlet. 2017.11.003

8 Gao A, Sun T and Ma G: LEM4 confers tamoxifen resistance to breast cancer cells by activating cyclin D-CDK4/6-Rb and ER $\alpha$ pathway. Nat Commun 9: 4180, 2018. PMID: 30301939. DOI: 10.1038/s41467-018-06309-8

9 Wang-Bishop L, Chen Z and Gomaa A: Inhibition of AURKA reduces proliferation and survival of gastrointestinal cancer cells with activated KRAS by preventing Activation of RPS6KB1. Gastroenterology 5085: 35161-35168, 2018. PMID: 30342037. DOI: $10.1053 /$ j.gastro.2018.10.030

10 Wei TW, Wu PY and Wu TJ: Aurora A and NF-kB survival pathway drive chemoresistance in acute myeloid leukemia via the TRAF-Interacting protein TIFA. Cancer Res 77: 494-508, 2017. PMID: 28069801. DOI: 10.1158/0008-5472.CAN-16-1004

$11 \mathrm{Wu}$ CC, Yu CT, Chang GC, Lai JM and Hsu SL: Aurora-A promotes gefitinib resistance via a NF-kB signaling pathway in p53 knockdown lung cancer cells. Biochem Biophys Res Commun 405: 168-172, 2011. PMID: 21216229. DOI: 10.1016/ j.bbrc. 2011.01 .001

12 Lan F, Yue X, Ren G, Li H, Ping L and Wang Y: miR-15a/16 enhances radiation sensitivity of non-small cell lung cancer cells by targeting the TLR1/NF-kB signaling pathway. Int J Radiat Oncol Biol Phys 91: 73-81, 2015. PMID: 25442346. DOI: 10.1016/j.ijrobp.2014.09.021

13 Jin R, Yi Y, Yull FE, Blackwell TS, Clark PE and Koyama T: NF-kB gene signature predicts prostate cancer progression. Cancer Res 74: 2763-2772, 2014. PMID: 24686169. DOI: 10.1158/0008-5472.CAN-13-2543

14 Wang LH, Yang JY, Yang SN, Li Y, Ping GF and Hou Y: Suppression of NF-kB signaling and P-glycoprotein function by gambogic acid synergistically potentiates adriamycin-induced apoptosis in lung cancer. Curr Cancer Drug Targets 14: 91-103, 2014. PMID: 24245692. DOI: 10.2174/15680096136661311 13100634

15 Wamsley JJ, Kumar M, Allison DF, Clift SH, Holzknecht CM and Szymura SJ: Activin upregulation by NF- $\mathrm{kB}$ is required to maintain mesenchymal features of cancer stem-like cells in nonsmall cell lung cancer. Cancer Res 75: 426-435, 2015. PMID: 25432175. DOI: 10.1158/0008-5472.CAN-13-2702

16 Tsuchiya Y, Asano T, Nakayama K, Kato T Jr. Karin M and Kamata $H$ : Nuclear IkB- $\beta$ is an adaptor protein for IкB- $\alpha$ ubiquitination and degradation in UV-induced NF-kB activation. Mol Cell 39: 570-582, 2010. PMID: 20797629. DOI: 10.1016/ j.molcel.2010.07.030

17 Keklikoglou I, Koerner C, Schmidt C, Zhang JD, Heckmann D and Shavinskaya A: MicroRNA-520/373 family functions as a tumor suppressor in estrogen receptor negative breast cancer by targeting NF-kB and TGF- $\beta$ signaling pathways. Oncogene 31 : 4150-4163, 2012. PMID: 22158050. DOI: 10.1038/onc.2011.571

18 Furusawa Y, Wei ZL, Sakurai H, Tabuchi Y, Li P and Zhao QL: TGF- $\beta$-activated kinase 1 promotes cell cycle arrest and cell survival of X-ray irradiated HeLa cells dependent on p21 induction but independent of NF-kB, p38 MAPK and ERK phosphorylations. Rad Res 177: 766-774, 2012. PMID: 22490 020. DOI: $10.1667 / \mathrm{rr} 2792.1$

19 Chuang HY, Lee YP, Lin WC, Lin YH and Hwang JJ: Fatty acid inhibition sensitizes androgen-dependent and -independent prostate cancer to radiotherapy via FASN/NF-kB Pathway. Sci Rep 9: 13284, 2019. PMID: 31527721. DOI: 10.1038/ s41598-019-49486-2

Received September 23, 2019

Revised October 18, 2019

Accepted October 24, 2019 